\title{
Complex Protostellar Chemistry
}

\author{
Joseph A. Nuth III and Natasha M. Johnson \\ Solar System Exploration Division \\ NASA's Goddard Space Flight Center \\ Greenbelt MD 20771
}

Two decades ago, our understanding chemistry in protostars was simple-matter either fell into the central star or was trapped in planetary-scale objects. Some minor chemical changes might occur as the dust and gas fell inward, but such effects were overwhelmed by the much larger-scale processes that occurred even in bodies as small as asteroids. The chemistry that did occur in the nebula was relatively easy to model because the fall from the cold molecular cloud into the growing star was a one-way trip down a well-known temperature pressure gradient; the only free variable was time. However, just over 10 years ago it was suggested that some material could be processed in the inner nebula, flow outward, and become incorporated into comets $(1,2)$. This outward flow was confirmed when the Stardust mission returned crystalline mineral fragments (3) from Comet Wild 2 that must have been processed close to the Sun before they were incorporated into the comet. In this week's Science Express, Ciesla and Sandford (4) demonstrate that even the outermost regions of the solar nebula can be a chemically active environment. Their finding could have consequences for the rest of the nebula.

Outward flow in the nebula is the natural result of conserving the total angular momentum of the star-forming system as mass continues to accrete onto the rotating disk feeding the growth of the central star (5-7). As mass flows inward along the flared accretion disk, additional mass flows slowly outward along the midplane (see the figure). As an additional complication, because the midplane is hotter than the top of the disk, convection will also mix materials vertically in the nebula. Ciesla and Sandford show that ice-coated dust grains, moving outward and subject to convection, will be exposed to cosmic radiation that is sufficient to cause the same chemical effects seen in dark cloud cores-that is, the conversion of simple carbon- and nitrogen-containing molecules into more complex organic species-and so will have consequences for nebular chemistry.

It has been nearly 40 years since the discovery that the oxygen in solid bodies throughout the solar system is mass-independently fractionated (8). It appears as if ${ }^{16} 0$ were added or subtracted from the bulk composition of the dust independently of either ${ }^{17} 0$ or ${ }^{18} 0$. While the first explanation for this observation was the addition of 160 -rich supernova grains to the forming solar system (8), the currently favored explanation is known as chemical selfshielding (9-11). The principle is similar to that of laser isotopic separation of gases, but uses either interstellar ultraviolet (UV) radiation or the light of the growing Sun. In either case, UV radiation dissociates $\mathrm{CO}$ molecules. However, owing to the abundance of $\mathrm{C}^{16} \mathrm{O}$ molecules, the radiation penetrating the system runs out of photons capable of breaking up this isotopic combination before it runs out of photons that dissociate $\mathrm{C}^{17} \mathrm{O}$ or $\mathrm{C}^{18} \mathrm{O}$. The result is that in such regions, ${ }^{17} 0$ and ${ }^{18} 0$ are free to react, either directly with dust grains 
or with $\mathrm{H}$ atoms to form $\mathrm{H}_{2} \mathrm{O}$ that eventually reacts with the dust. Meanwhile, the extremely stable $\mathrm{C}^{16} \mathrm{O}$ sequesters the ${ }^{16} \mathrm{O}$ in the gas phase and prevents it from reacting with silicates. As long as $\mathrm{CO}$ remains stable, the self-shielding mechanism remains a viable explanation for the mass-independent fractionation of oxygen isotopes throughout the solar system.

Unfortunately, Ciesla and Sandford demonstrate that CO is not a stable sink for ${ }^{16} 0$, and that such molecules will be converted into complex organics even in the cold outer reaches of the solar nebula, just as they are in cold molecular cloud cores. If $\mathrm{CO}$ is converted into organic molecules by radiation processing in icy grains in the outer nebula, just as it is via Fischer-Tropsch-type reactions (12-14) on the surfaces of warmer grains in the inner nebula, and if such organics are free to react with silicate dust or to react with the bulk silicate in planetesimals, then the effects of photochemical self-shielding will be minimized or even completely erased. A new mechanism may be needed to explain the oxygen isotopic fractionation observed in the solid bodies of the solar system.

Our understanding of the chemistry in protostellar systems has made enormous progress over the last few decades, fueled by an increased awareness of the complex dynamics of these evolving energetic nebulae. We can no longer consider just the simple local environment (15) to explain the composition of a planet, asteroid, or comet as was done in the past, but must now consider chemical processes that might take place within the nebula as a whole as well as the probability of transport and mixing the products of such reactions throughout the system. Just as we now find it impossible to explain the complex chemistry of the terrestrial atmosphere without reference to detailed transport models that interconnect highly dissimilar chemical environments, so chemical models of protostars and of the solar nebula must eventually treat these environments as tightly coupled, interactive systems. The demonstration that the chemistry on the surfaces of outwardflowing, dynamically mixing icy grain surfaces both mimics the chemistry in cold cloud cores and strikes at the central assumption of the photochemical self-shielding model for oxygen isotopes in solar system solids only adds emphasis to this conclusion.

\section{References}

1. J. A. Nuth, H. G. M Hill, G. Kletetschka, Nature 406, 275 (2000).

2. J. A. Nuth, Am. Sci. 89, 230 (2001).

3. M. Zolensky et al., Science 3141735 (2006).

4. F. J. Ciesla, S. A. Sandford, Science 336, XXX (2012); 10.1126/science.1217291.

5. F. J. Ciesla, Science 318, 613 (2007).

6. F. J. Ciesla, Meteorit. Planet. Sci. 43, 639 (2008).

7. F. J. Ciesla, Icarus 200, 655 (2009).

8. R. N Clayton, L. Grossman, T. K. Mayeda, Science 182, 485 (1973).

9. H. Yurimoto, K. Kuramoto, Science 305, 1763 (2004).

10. J. R. Lyons, E. D. Young, Nature 435, 317 (2005).

11.R. N. Clayton, Nature 415, 860 (2002).

12. R. Hayatsu E. Anders, Top. Curr. Chem. 99, 1 (1981).

13. J. Llorca, I. Casanova, Meteorit. Planet. Sci. 33, 243 (2000).

14. J. A. Nuth, N. M. Johnson, S. Manning, Astrophys. J. 673, L225 (2008).

15. J. S. Lewis, Sci. Am. 230, 50 (March 1974). 


\section{Figure 1.}

Large scale motion driven by conservation of angular momentum, together with more local convective cells above and below the hotter nebular mid-plane dynamically mix products of chemical reactions from many different environments throughout the nebula.

(graphic design depiction of transport/movement within the solar nebula) 